\section{Die Sicht des Täters}

Den Autorinnen des hier vorgestellten Buches ist es gelungen, sich dem Thema ,Sexueller Missbrauch“ unter Berücksichtigung verschiedener Perspektiven anzunähern. Dabei werden juristische, soziale und psychologische Aspekte angesprochen. Damit ist das Buch ein umfassendes Nachschlagewerk für verschiedene Berufsgruppen.

Die Bearbeitung des Themas aus der Perspektive der Täter ist spannend und überaus wichtig. Die Autorinnen haben erfolgreich die Hintergründe für eine solche Tat beleuchtet, ohne diese zu verharmlosen oder die Opfer zu diffamieren. Sehr interessant ist das Kapitel zu den Tätertypologien. Hier werden aus verschiedenen Blickrichtungen - nach einer forensischen, einer sozialen und einer psychologischen Einteilung - unterschiedliche Typologien vorgestellt. Insgesamt gelingt es gut, die Bedeutsamkeit des lebensgeschichtlichen Hintergrunds der Täter (eigener Missbrauch, Gewalterfahrungen etc.) herauszuarbeiten.
Das Buch ist gut lesbar und systematisch aufgebaut. Jedes Kapitel ist in sich geschlossen, so dass der Leser die Möglichkeit hat, das Buch chronologisch zu lesen oder aber einzelne Kapitel einzeln herauszugreifen. Die sich dadurch ergebenen Redundanzen sind vor dem Hintergrund der Komplexität des Themas eher hilfreich als störend.

Jessica Spaeth, Dario Arconada Valbuena

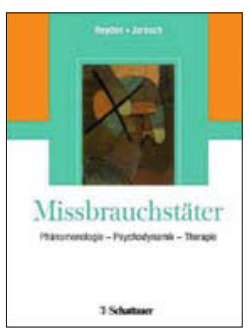

Saskia Heyden, Kerstin Jarosch Missbrauchstäter Schattauer Verlag, Stuttgart 2009 $39,95 €$ ISBN 978-3-7945-2633-8

\section{Immer up to date}

Die 3. Auflage des „Speer/Gahr" wurde vollständig neu bearbeitet und mit fast 700 überwiegend farbigen Abbildungen und über 400 Tabellen großzügig ausgestattet. Auf 1.100 Seiten ist jetzt die gesamte Pädiatrie in 16 Kapiteln umfassend dargestellt. Im Sonderkapitel „Pädiatrische Spezialbereiche“ sind Gynäkologie, Orthopädie, Chirurgie, Augenheilkunde, HNO, Dermatologie und Zähne sowie die Sportmedizin bei Kindern für die Praxis ausreichend vertreten.

Das hervorragend modernisierte Buch richtet sich an Studenten, junge angehende Stationsärzte und Kollegen anderer Fachrichtungen, die Kinder mitbehandeln. Aber auch „gestandene“ Fachärzte werden an diesem übersichtlichen Werk ihre Freude haben und fast auf jeder Seite neue Aspekte der Pädiatrie entdecken.

Dr. Friedrich Grüttner

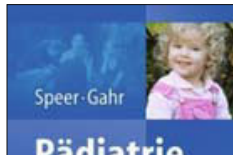

Christian P. Speer, Manfred Gahr Pädiatrie Pädiatrie Springer Medizin, Heidelberg 2009 $99,95 €$ ISBN 978-3-54069479-3 